\title{
Antistreptolysin O (ASO) titers and beta-haemolytic streptococcus (BHS) in Children in Calabar, Nigeria.
}

\author{
Mandor Baki I ${ }^{1}$, Asuquo Anne $\mathrm{E}^{2}$, Abia-Bassey L $\mathrm{L}^{2},{ }^{3}$ Benjamin Thumamo P, \\ ${ }^{4}$ Akpan I A Etuk I $S^{5}$, Eyong $\mathrm{M} \mathrm{E}^{5}$, Meremiku $\mathrm{M} \mathrm{M}^{5}$ \\ ${ }^{I}$ (Department of Microbiology and parasitology, University of Calabar Teaching Hospital, Calabar, Nigeria) \\ ${ }^{2}$ (Department of Medical Laboratory Science, College of Medical Sciences University of Calabar, Nigeria) \\ ${ }_{3}^{3}$ (Department of Medical Laboratory Science, Faculty of Health Sciences, University of Buea, Cameroon) \\ ${ }^{4}$ (Department of Family medicine, People's Clinic, Calabar,Nigeria) \\ ${ }_{5}^{5}$ (Department of Paediatrics College of Medical Sciences, University of Calabar, Nigeria)
}

\begin{abstract}
This study investigated the prevalent ASO titer values and serogroups of BHS prevalent in the throat of apparently healthy children in Calabar with the aim of detecting the risk in the children of developing RF and $R H D$ in their later years.

The throats of 437 children drawn randomly from elementary schools in three communities in Calabar, Nigeria were examined and throat swabs collected. Fifty children clinically diagnosed with bacterial pharyngitis were used as control. Throat swabs were cultured aerobically on goat blood agar in an atmosphere of 5-10\% carbon-dioxide and grouped serologically using the Strep PRO grouping kit. ASO titers in the sera of 437 subjects were determined by the Rheumajet ASO 500T. Culture of the 437 throat specimens yielded 90(20.6\%) Streptococcal species belonging to 5 groups, 4 of which were definite and included $C, D, F$ and $G$. The fifth group was not identified by the available grouping kit. The most prevalent Streptococcus group in the study was group C with 32(35.6\%) isolates. The most frequently occurring ASO titer was 400IU/mL, obtained from 186/437(42.5\%) subjects, with those in Group C associated with high titers (30; 93\%). Overall, the mean ASO titers for test and control subjects were $902 I U / m L$ and $929 I U / m L$ respectively. There was a positive correlation ( $r=0.5, P=0.000$ ) between presence of Streptococci in the throats and high ASO titers in the sera of subjects.
\end{abstract}

The study established a high prevalence of BHS in the throat of children in Calabar howbeit, with no evident risk of RF and RHD as no Group A Streptococcus was isolated.

Keywords: beta-haemolytic streptococci (BHS), rheumatic fever (RF), rheumatic heart disease (RHD), Antistreptolysin $O$ antibodies (ASO), pharyngitis

\section{Introduction}

Rheumatic heart disease (RHD) and rheumatic fever (RF) remain significant causes of cardiovascular disease and mortality in children particularly in the developing countries [1]. RF is estimated to affect 20 million people and is a leading cause of death during the first five decades of life [2]. It is endemic in developing countries with annual incidence of 100-200 cases per 100,000 school aged children [(3]. Particularly frustrating has been the fact that RF and RHD are theoretically preventable but still remains a problem in these countries. Reason most advanced is that these countries cannot afford the economic and social cost involved [4, 1]. $\mathrm{RF}$ is an auto immune consequence of infection of the throat (phryngitis) with Group A haemolytic Streptococci (GAS) which causes an acute generalized inflammatory response and an illness that selectively affects the heart, joints, brain and skin [5].

$\mathrm{RF}$ leaves no lasting damage to the brain, joints or skin. However, damage to the heart valves, particularly the mitral and aortic valves, may persist 2-3weeks after an acute episode has resolved. This involvement of the cardiac valves is known as Rheumatic Heart Disease (RHD), the most significant sequela of ARF $[1,5,6]$.

The beta haemolytic streptococci group $\mathrm{C}$ and $\mathrm{G}$ have also been reported as causing acute pharyngitis but their involvement in RF and RHD is still controversial [7,8,9,10]. GAS infection is spread by droplets, thus accounting for it high transmissibility in such situations [11]. Most outbreaks are associated with respiratory tract infection, but food-borne outbreaks often with dairy products and eggs have been documented [12, 13, 14].

GAS infections have a unique nature in that; there are more than 150 recognized $\mathrm{M}$ - serotypes that can cause the infections in contrast to other infectious agents due only to a single serotype. Although infection with a single serotype is thought to confer long lasting type-specific immunity, the abundance of the serotype makes the threat of a new infection continuous [1]. Therefore, People who have had RF previously are much more likely to have subsequent episodes, and these recurrences may cause further damage to the cardiac valves. Thus RHD steadily worsens in people who have multiple episodes of R F $[1,5,6]$. Understanding the epidemiology 
and pathogenesis of GAS infections associated with RF is the fact that some specific serotypes appear to have more "rheumatogenic" potentials than others. Epidemiological observations suggest that M types 1, 3, 5, 6, 18, 24 , are associated with RF and are most often found to infect the upper respiratory tract. In contrast superficial skin infection serotypes are among the higher numbered M-types (M-types 49, 55, 57, 60) and are not associated with RF [1]. Early diagnosis and adequate treatment of GAS infection is an important initial stage in primary prevention of RF.

The diagnosis of RF is not usually possible through recovery of streptococci from throat swab cultures alone as it may indicate acute infection or a carrier state seen in $2.5-35.4 \%$ of individuals [15]. Moreover positive throat cultures are obtained in only about $11 \%$ individuals at the time of presentation of RF [16]. Therefore, evidence of host immune response to GAS antigens is required to confirm the diagnosis. The assay for ASO is widely used [17], although it has been shown to be non-specific. This is because antibodies to streptolysin are produced by both group A and non group A streptococci particularly the C and G groups [18].

There are well documented data on prevalence, serogroups of BHS and ASO titres levels in asymptomatic carriers elsewhere in the world $[19,20,21]$ but there is a dearth of such data in Calabar, Nigeria. Consequently, this study was aimed at establishing for the first time such data with the hope of identifying those at risk of developing RF.

\section{Materials And Methods}

The four hundred and thirty seven subjects investigated in this study aged 3-15years (196males and 241females) without any apparent throat or respiratory infection were drawn from elementary schools in Calabar. Blood was collected through veni-puncture from all subjects involved in the study for ASO determination.

Subjects who had received antibiotics 2-5days prior to sample collection were excluded from the study.

Fifty children clinically diagnosed with tonsillitis, pharyngitis or tonsillopharyngitis served as control. The study took place between June 2009 and June 2010. Throats of all subjects were examined by a paediatrician prior to collection of throat swabs.

Throat swabs collected with the aid of a tongue depressor were cultured aerobically on 5\% goat blood agar medium, incubated at $37^{\circ} \mathrm{C}$ under $5-10 \% \mathrm{CO}_{2}$ for $24-48 \mathrm{hrs}$. Colonies were identified by their gram's reaction, biochemical tests and haemolytic patterns on blood agar.

Serogrouping of the overnight purified isolates was done by the Strep PRO ${ }^{\mathrm{TM}}$ Grouping kit (Hardy diagnostic, India). This Grouping kit provides a rapid latex agglutination method for the serological identification of Lancefield's groups A, B, C, D, F, and G from isolated colonies of beta-haemolytic Streptococcus species.

Anti-streptolysin O (ASO) levels in the blood of all the subjects was determined using the Rheumajet $^{\mathrm{TM}}$ ASO 500T(Biokit USA, Inc).This is a rapid qualitative and semi-quantitative determination of ASO titer in serum by agglutination of the latex particles on slide.ASO titers for this study were determined semi-quantitatively. Both tests were carried out according to manufacturers`instructions.

\section{Results}

A total of 90 (20.6\%) $\beta$-haemolytic streptococci belonging to 4 Lancefield serogroups were isolated from the throats of the 437 test subjects. The distribution of BHS and ASO titers of test subjects by age is shown in fig 1. ASO titers as well as BHS throat colonization of test subjects correlated positively with age. The lowest BHS isolation rate was seen among children <6yrs of age. Isolation rate increased with age group and peaked in age group 10-12yrs then declined in age group 13-15yrs. The mean ASO titers per age group followed a similar pattern to that of BHS isolation and age group.

BHS serogroup distribution by gender is shown in fig 2. Group C BHS were the most isolated in both males (15) and females (17) while group D were the least isolated BHS serogroup males (3) and females (2). UID were isolated more in females (16) than in males (6).

The distribution of isolates by Lancefield grouping is as follows: group C 32(35.6\%), group F 17(18.9\%), group G 14(15.6\%) and group D 5(5.6\%). Twenty two (24.4\%) of the isolates did not match any of the known Lancefield groups with the available antisera and were designated as the unidentified group (UID). From the 50 control subjects, 30 BHS isolates were obtained and included group A 4 (13.3\%), group B 1(3.3\%), group C 19(63.3\%), group F 2(6.7\%) and group G 4(13.3\%) The control subject in whom BHS group B was isolated from and one from whom BHS group $\mathrm{C}$ was isolated from refused blood collection (TABLE 1).

The ASO titers in 437 test and 47 control subjects are shown on TABLE 2 . Three hundred and thirty (75.5\%) of the test subjects had ASO titers $\leq 400 \mathrm{IU} / \mathrm{ml}$ and $16(4.8 \%)$ harbored BHS, 11 of which were UIDs. Nineteen $(40.4 \%)$ of the control subjects had ASO titers $\leq 400 \mathrm{IU} / \mathrm{ml}$ and $1(5.3 \%)$ harbored BHS. The most frequently occurring titers in the study were $400 \mathrm{IU} / \mathrm{ml}$ in $186(42.6 \%)$ test and $800 \mathrm{IU} / \mathrm{ml}$ in $22(46.8 \%)$ control subjects. The mean ASO titer obtained from the test and control groups were $446 \mathrm{IU} / \mathrm{ml}$ and $766 \mathrm{IU} / \mathrm{ml}$ 
respectively. However, the mean ASO titer for 90 asymptomatic carriers in the test group was $902 \mathrm{IU} / \mathrm{ml}$ while that for the 28 control subjects from whom BHS isolates were obtained was $929 \mathrm{IU} / \mathrm{ml}$. There was no significant difference ( $\mathrm{p}=0.4622)$ between the two means.

There was a positive correlation $(\mathrm{r}=0.5468 \mathrm{p}=0.000)$ between high ASO titers and occurrence of BHS in throats of subjects. Specifically, 59/90 (65.6\%) and 22/28 (78.6\%) of the BHS isolates respectively in test and control subjects were associated with ASO titer of 800IU/ml (TABLE 2).

The most predominant serogroup in this study for test and control subjects was $\mathrm{C}$ and mainly associated with titers of $800 \mathrm{IU} / \mathrm{ml}$ and $1600 \mathrm{IU} / \mathrm{ml}$ (Table 1). The UIDs, present only in the test group, cut across all the ASO titers (TABLE 1), and were more prevalent in females 16 (72.7\%) than males 6 (27.3\%) (Fig.2). The four Group A streptococci were isolated from control subjects, three were obtained from subjects with ASO titer of $1600 I U / m l$ (TABLE 1).

\section{Discussion}

The $20.6 \%$ prevalence of $\beta$-haemolytic streptococci in the throat of children in Calabar was high compared to a previous study (13.3\%) in Lagos, Nigeria [22]. [20] reported 21.4\% in Chennai, India while $15.4 \%$ was reported in the Tri-Island State of Grenada [19]. Factors affecting BHS prevalence among healthy school aged children include geographic location, socio-economic instability, overcrowding among other environmental factors $(23,24]$.

BHS and ASO titers in this study increased with age group of subjects and peaked at age group 1012 years. This is consistent with previous studies although GAS and not non GAS were involved [25, 26]. Other studies had demonstrated the same age- associated rise in ASO but had differing peak age groups. [27] observed a peak at age 9 and 10years while [21] observed a peak at age 11-15 years. The differences observed could be explained by fact that ASO levels in children may be influence by the study population, climatic conditions and other factors [21].

Neither ASO titer nor BHS throat colonization was influenced by gender of subjects.

This finding is similar to that of [28] who had reported that tonsillitis affects both sexes equally.

Predominance of group C in both test and control subjects suggests its involvement in tonsillitis in the study area. Group $\mathrm{C}$ and $\mathrm{G}$ Streptococci are reportedly common causes of tonsillitis or pharyngitis in both adults and children in tropical countries [8, 9, 11] while group A is common in the temperate countries [29]. Other streptococcal groups of $\mathrm{D}, \mathrm{F}$ and $\mathrm{G}$ isolated in this study have previously been implicated in tonsillitis [30]. [31] isolated streptococci groups A, B, C, G and a group called non-group A, B, C, D. This latter group may be likened to the UID group in our study. The high carriage of non group A streptococci has been reported elsewhere [32] The high carriage of group C and $\mathrm{G}$ is of great concern especially now that there has been increasing report of their involvement in human throat infections, other pyogenic infections [10] and in RF [7].

The uncommon finding of the absence GAS carriage could be attributed to the number of subjects /community studied and possibly low carriage during the period of study. However, [33] had a similar finding although their subjects were older (average age of 22.6yrs).

The predominance of the ASO titers of $\leq 400 \mathrm{IU} / \mathrm{ml}$ in up to $75.5 \%$ of test subjects with only $4.8 \%$ isolation of BHS may suggest that these titers are neither associated with past infection nor recent. This may be suggestive of a baseline titer but more work needs to be carried out to ascertain this.

The comparable mean ASO titers of $902 \mathrm{IU} / \mathrm{ml}$ and $929 \mathrm{IU} / \mathrm{ml}$ in asymptomatic carriers in the test group and symptomatic (control subjects) corroborates the assertion by [34] that carriage of BHS is associated with high ASO titers. The mean of test subjects could have been influenced by the two subjects who had a titer of $3200 \mathrm{IU} / \mathrm{ml}$. A follow up of this children was not possible because the schools vacated soon after the sample were collected and even when they resumed the parents refused any further investigation on the children.

The positive correlation between ASO titer and isolation of BHS in culture in this study may not be unconnected with uncomplicated streptococcal infections as positive throat cultures are obtained in only about $11 \%$ individuals at the time of presentation of RF [16].

\section{Conclusion}

This study found a high prevalence(20.6\%) of BHS in the throat of apparently healthy children in Calabar with group C identified as the most common streptococcal group. However, four GAS were isolated from control (symptomatic) subjects. ASO titer of $400 \mathrm{IU} / \mathrm{ml}$ may be the baseline titer for this population. Other studies previously reported baseline ASO titer of less than 400IU/ml. This would prove helpful in the interpretation of elevated ASO titers in cases of suspected RF and acute glomerulonephritis. The risk of ARF and RHD based on GAS carriage in the throat of the children was not apparently from this study. 


\section{Acknowledgement}

The authors thank the coordinator Microbiology/parasitology laboratory for allowing us access to the laboratory where the laboratory aspect of the work was carried out. For their contribution in the work the authors are grateful to the administrators of the schools who accepted to participate in the study. We are thankful to the parents/guardians of children for permitting us to use their children.

\section{References} pharyngitis, AHA Scientific statement Endorsed by American Academy of Pediatrics Circulation, 119, 2009, 1541-51. J. Carapetis, A. Steer, E. Mulholland, et al. The Global Burden of Group A Streptococcal Disease, Lancet Infectious Diseases, 5 , 2005, 685-694.

[3] M. Batzloff, H. Yan, M. Davies, et al. Preclinical Evaluation of a Vaccine Based on Conseved Region of M protein that prevents group A Streptococcal Infection, Indian Journal of medical Research, 119suppl, 2004, 104-107.

[4] E. H. Ismail, Rheumatic fever, Bailliere's Clinical Rheumatology, 9, 2006, 11-120.

[5] R.M. Manase, Awareness of Rheumatic Heart Disease Prevention among Primary Health care Providers and People aged 9years and above in Kinondoni Municipality Cares Salam, Tanzania. Muhimbili University of Health Allied Science (Doctorate Dissertation) 2011.

[6] R. K. Krishna and R. Tondon, Rheumatic Fever and Rheumatic Heart Disease: The last 50years, Indian Journal of Medical Research, 137, 2013, 643-658.

[7] A. Haiden, S.R. Talay, M. Rohde, et al. Pharyngeal carriage of Group C and Group G streptococci and acute rheumatic fever in an Aboriginal population, Lancet, 356, 2000, 1167-1169.

[8] M. A. Zaoutis, R. Gross, and V. Klein, The role of group C and group G streptococci in acute pharyngitis in children, Clinical Microbiology Infection, 10, 2004, 37-40.

[9] S. Hashikawa, Y. Iinuma,and T. Furushita, Characterization of group C and group G streptococcal strains that cause streptococcal toxic shock syndrome, Journal of Clinical Microbiolology, 42, 2004, 186-192.

[10] T. Menon, S. Shanmugasundaram, M.P. Kumar, et al. Group A streptococcal infections of the pharynx in a rural population in south India, Journal of Medical Research, 119, 2004, 171-173.

[11] S. Aamir, A. Muhammed, M. lbrahim, et al. Pharyngitis and Sore Throat: (review), African Journal of Biotechnology, 10, 2011, 6190-6197.

[12] M. Levy, J. Christine, Ed Kraa, Tonsillopharygitis Caused by Foodborne Group A Streptococcus: A Prison- based outbreak, Journal of Royal Society of Tropical Medcine and Hygiene. 36(2), 2011, 175-182.

[13] G. Falkenhorst, J. Bagdanaite, M. Lisby, et. al. Outbreak of Strep Throat Infection: Don't forget to ask about food, Epidemiology and Infection, 136, 2008, 1165-1171.

[14] A.J, Painter, R.M. Hoekstra, and T. Ayers, Attribution of Food borne illnesses, Hospitalization and Deaths to Food Commodities by using Data, United States, 1998-2008, Emerging Infectious Diseases, 19(3), 2013, 407-415.

[15] M.E.Pichichero, S.M. Marsocci, M. Murphy, et al. Incidence of streptococcal carriers in private practice. Archives of Pediatrics \& Adolescent Medicine, 153, 1999, 624-28.

[16] E.L. Kaplan, Rheumatic Fever, Harrison' Principle of Internal Medicine, (New York: McGraw-Hill, 2001)

[17] A.K. Alyaa, M.H. Nevin, and E.E. Sahar, Antistreptolysin O titer in health and disease: levels and significance, Pediatric Reports, 4, 2012, 1- 8.

[18] L. Johanson, and N. Mansson, Rapid test, throat culture, and Clinical assessment in the diagnosis of tonsillitis, Family Practice,20, 2002, 108-111.

[19] T. Noel, J. Zabriskie, C. Macpherson, et al. Beta-haemolytic Streptococci in school children 5-15 years with an emphasis on rheumatic fever, in the tri-island state of Grenad, West Indian Medical Journal, 54, 2005, 22-27.

[20] A.Charmaine, E. Swama, and M.Thangam, Pharyngeal carriage of group A Streptococcus in school children in Chennai, Indi, India Journal of Research, 124, 2006, 195-8.

[21] S.Sunil, K. Kirti, K. Aushik, et al. Anti-streptolysin O antibodies in normal healthy children 5-15 years, Indian Pediatrics, 40, 2003, 1068- 1071.

[22] S.F. Lawal, A.O. Coker, E.O. Salanke, et al. Lancefield Group G streptococci in Nigeria, Journal of Medical Microbiology, 15, 1987, 123-25.

[23] R.Gonzales, D. Malone, J. Maselli, et al. Excessive antibiotic use for acute respiratory infections in United States, Clinical Infectious Disease, 33, 2001, 757-762.

[24] B. Amal, B. Shahira, E. Gamal, et al. Identification of Clinical Criteria for Group A beta- haemolytic streptococcal pharyngitis in children living in a rheumatic fever endemic area. Journal of tropical paediatrics, 48, 2002, 285-93.

[25] S. Dumre, K. Sapkota, N. Adhikari et al. Asymptomatic throat carriage rate and antimicrobial resistence pattern of streptococcus. Medica, 17, 2009, 392-396.

[26] E.L. Kaplan, D.R.Constance, and R.J. Dwight, Antistreptolysin O and anti-deoxyribonuclease B titers: normal Values for children ages 2 to 12 in the United State, Pediatrics, 101, 1998 86-88.

[27] P.D. Gunatillake and T.D. Perera, Antistreptolysin O Titres amongst Children in a Rural Area of Ceylon, The Journal of Hygiene, 68, 1970, 13-17.

[28] J.T. Benoy, Robert DP, Michael T L, Pharyngitis, Bacterial eMedicine, 2006, 1-12.

[29] A. A. Mostafa, E B. Naser, A B. Ibrahim et al, Throat culture screening for beta-haemolytic streptococci among schoolboys in Saudi Arabia, Eastern Mediterranean Health Journal, 3, 1996, 425-31.

[30] J. Begovac, E Bobinac, B Benic et al, Asymptomatic pharyngeal carriage of beta-haemolytic streptococci and streptococcal pharyngitis among patients at an urban hospital in Croatia, European Journal of Epidemiology, 9, 2004, 405-10.

[31] K. Seon-Ju, Bacteriological characterization and serotyping of Streptococcus pyogenes isolated from throats of school children, Yonsei Medical Journal 2000; 41: 56-60.

[32] K. Lakshmana, A. Mohammed M, S R. Bindu Rani et al, High Throat Carriage of Groups G/C streptococci among School Children in Bangalore City and its significance, African Journal of Microbiology Research, 6, 2012, 7128-7130.

[33] J. C. Turner, F G. Hayden and M C. Lobo, Epidemiologic evidence for Lancefield group C beta-hemolytic streptococci as a cause of exudative pharyngitis in college student, Journal of Clinical Microbiology, 35, 1997, 1-4.

[34] E. Kaplan, The Group A Streptococcal upper respiratory tract carrier state: enigma, Journal of Pediatric, 97, 1980, 337-345. 


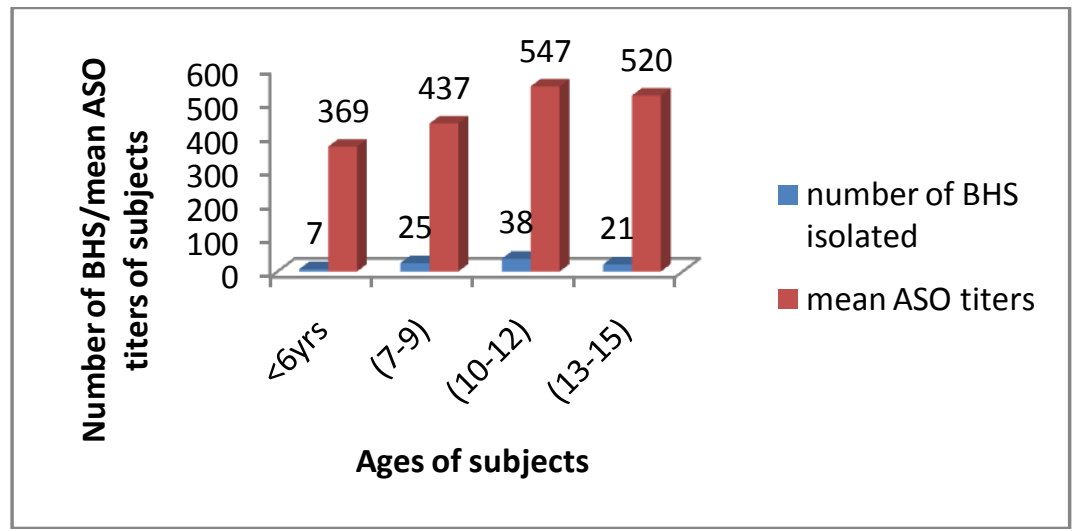

figure1: Distribution of BHS and ASO titers of test subjects by age

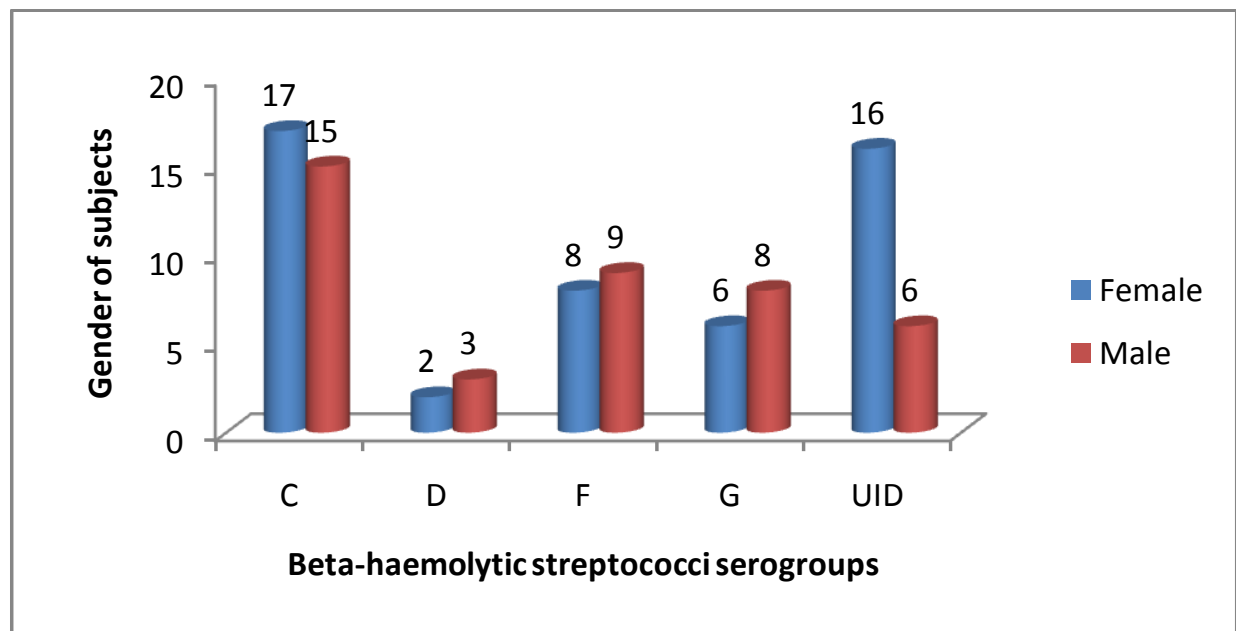

figure 2: Distribution of BHS serogroups by gender

Table 1: Association between ASO titres and BHS Lancefield groupings of test and control subjects

\begin{tabular}{|c|c|c|c|c|c|c|c|c|c|}
\hline \multirow{2}{*}{$\begin{array}{l}\text { ASO } \\
\text { titers(UI/ml)/Subject } \\
\text { group }\end{array}$} & \multicolumn{5}{|c|}{$\begin{array}{l}\text { Test subjects } \\
\text { Number of isolates per Lancefield grouping }\end{array}$} & \multicolumn{3}{|c|}{$\begin{array}{l}\text { Control subjects } \\
\text { Number of isolates per } \\
\text { grouping }\end{array}$} & Lancefield \\
\hline & $\mathrm{C}$ & $\mathrm{D}$ & $\mathrm{F}$ & $\mathrm{G}$ & UID & $\overline{\mathrm{A}}$ & $\mathrm{C}$ & $F$ & $\mathrm{G}$ \\
\hline$<200$ & 0 & 0 & 0 & 0 & 2 & 0 & 0 & 0 & 0 \\
\hline 200 & $\overline{0}$ & $\overline{0}$ & $\overline{0}$ & $\overline{0}$ & 2 & $\overline{0}$ & $\overline{0}$ & $\overline{0}$ & 0 \\
\hline 400 & 2 & $\overline{0}$ & 2 & 1 & 7 & $\overline{0}$ & $\overline{1}$ & $\overline{0}$ & 0 \\
\hline 800 & 19 & 5 & 15 & 11 & 9 & 1 & 15 & 2 & 3 \\
\hline 1600 & $\overline{11}$ & $\overline{0}$ & $\overline{0}$ & 2 & 1 & 3 & $\overline{2}$ & $\overline{0}$ & 1 \\
\hline 3200 & 0 & 0 & 0 & 0 & 1 & 0 & 0 & 0 & 0 \\
\hline Total & 32 & 5 & 17 & 14 & 22 & 4 & $18^{*}$ & 2 & 4 \\
\hline
\end{tabular}

*One subject did not allow blood collection

Table 2: Relationship between ASO titres and BHS of test and control subjects

\begin{tabular}{lll}
\hline $\begin{array}{l}\text { ASO titers } \\
\text { (IU/ml)/Subjects }\end{array}$ & $\begin{array}{l}\text { Test subjects } \\
\text { Number positive for ASO(BHS) }\end{array}$ & $\begin{array}{l}\text { Control subjects } \\
\text { Number positive for } \\
\text { ASO(BHS) }\end{array}$ \\
\hline$\frac{<200}{200}$ & $\frac{60(2)}{84(2)}$ & $\frac{5(0)}{8(0)}$ \\
$\frac{400}{800}$ & $\frac{186(12)}{87(59)}$ & $\frac{6(1)}{22(22)}$ \\
\hline$\frac{18(14)}{2(1)}$ & $\frac{5(5)}{1(1)}$ \\
\hline
\end{tabular}

